\title{
The Time Dependent Correlation Function of an Impenetrable Bose Gas as a Fredholm Minor. I
}

\author{
V. E. Korepin and N. A. Slavnov \\ Institute for Theoretical Physics, SUNY at Stony Brook, NY 11794-3840, USA
}

\begin{abstract}
We study the two field correlator of an Impenetrable Bose gas. Lenard [1] proved that the equal time correlator can be represented as a Fredholm minor. We generalize this representation to the time dependent correlator.
\end{abstract}

\section{Introduction}

We discuss the Bose gas in one space plus one time dimensions. The Hamiltonian [2] of the model is

$$
H=\int_{-L / 2}^{L / 2} d x\left(\partial_{x} \psi^{+} \partial_{x} \psi+c \psi^{+} \psi^{+} \psi \psi-h \psi^{+} \psi\right)
$$

Here $\psi(x)$ and $\psi^{+}(x)$ are canonical Bose fields: $\left[\psi(x), \psi^{+}(y)\right]=\delta(x-y)$, and $L$ is the volume. Only the case of an impenetrable boson is dealt with below, in this case the coupling constant $c=\infty$. The thermodynamics of the Bose gas was given in [3]. The chemical potential $h$ determines uniquely the density $D$. In [1] Lenard gives a representation of the time independent correlator $\left\langle\psi\left(x_{2}\right) \psi^{+}\left(x_{1}\right)\right\rangle$ as a Fredholm minor; this representation was used to write a differential equation for the correlator at zero temperature in [4]. The differential equation for the finite-temperature correlator was constructed in [5]. We can treat the Fredholm determinant obtained in this paper as a Gelfand-Levitan operator for some new differential equation, which describes the time dependent field correlator of the impenetrable Bose gas. The correlation function (Fredholm determinant) is the $\tau$-function of this new differential equation. We shall present this differential equation in the next publication.

Eigenfunctions of the Hamiltonian (1.1) (at $c=\infty$ ) were constructed in [2].

$$
\begin{aligned}
H\left|\Psi_{N}\right\rangle & =E\left|\Psi_{N}\right\rangle ; \quad\left|\Psi_{N}\right\rangle=\left|\Psi_{N}(\{\lambda\})\right\rangle, \\
\left|\Psi_{N}\right\rangle & =\frac{1}{\sqrt{N !}} \int d^{N} z \chi_{N}\left(z_{1}, \ldots, z_{N} \mid \lambda_{1}, \ldots, \lambda_{N}\right) \psi^{+}\left(z_{1}\right) \cdots \psi^{+}\left(z_{N}\right)|0\rangle .
\end{aligned}
$$


Here

$$
\chi_{N}=\frac{1}{\sqrt{N !}} \prod_{N \geqq j>k \geqq 1} \varepsilon\left(z_{j}-z_{k}\right) \sum_{p}(-1)^{[p]} \exp \left(i \sum_{n=1}^{N} z_{n} \lambda p_{n}\right) .
$$

$\varepsilon$-is the sign function, $P$ is a permutation and $\lambda_{j}$ are moments. Periodic boundary conditions can be written in the form

$$
e^{i L \lambda_{j}}=(-1)^{N-1} \text {. }
$$

The norm of the wave function is given by

$$
\left\langle\Psi_{N} \mid \Psi_{N}\right\rangle=\left\|\chi_{N}\right\|^{2}=L^{N}
$$

Let us denote the set of $\{\lambda\}$ in the ground state by $\left\{\mu_{j}\right\}$. For convenience, we take $N$ even. At zero temperature

$$
e^{i L \mu_{j}}=(-1)^{N-1}=-1, \quad \mu_{j}=\frac{2 \pi}{L}\left(j-\frac{N+1}{2}\right), \quad j=1, \ldots, N .
$$

In the thermodynamic limit $N \rightarrow \infty, L \rightarrow \infty$ (with the density $D=N / L$ fixed) the set of $\left\{\mu_{j}\right\}$ fill the interval $[-q, q]$ (Fermi-sphere) with

$$
q=\sqrt{h} ; \quad D=\frac{q}{\pi} .
$$

The value $q$ is called the fermi momentum. At finite temperature $T>0$ the distribution of particles in momentum space is given by the function $\rho\left(\mu_{j}\right)$ [3]:

$$
\rho(\mu)=\frac{1}{2 \pi}\left(1+e^{\left(\mu^{2}-h\right) / T}\right)^{-1} .
$$

We shall calculate the time-temperature correlator. The plan of the paper is as follows. In Sect. 2 the field form factor is represented as a matrix minor. In Sect. 3 the time correlator is calculated at zero temperature. In Sect. 4 the time temperature correlator is calculated. Appendix A contains calculations of singular sums. Appendix B gives a comparison with the Lenard formulae.

\section{Form Factor}

Let us consider the field form factor:

$$
F(x)=\left\langle\Psi_{N+1}\left(\{\lambda\}\left|\psi^{+}(x)\right| \Psi_{N}(\{\mu\})\right\rangle .\right.
$$

Here the set $\{\lambda\}$ consists of $N+1$ (odd) elements. So the equations for $\lambda$ and $\mu$ are different

$$
e^{i L \lambda_{J}}=(-1)^{N}=1 ; \quad e^{i L \mu_{j}}=(-1)^{N-1}=-1,
$$

and it follows that the $\lambda_{j}$ will never coincide with any of the $\mu_{k}$. The momenta $\lambda_{j}$ are equal to $2 \pi j / L(j \in Z)$,

$$
\lambda_{j}=\frac{2 \pi j}{L}
$$


The form factor (2.1) can be expressed in terms of the functions $\chi_{N}$ defined by (1.3):

$$
F(x)=\sqrt{N+1} \int d^{N} z \bar{\chi}_{N+1}\left(z_{1}, \ldots, z_{N}, x \mid\{\lambda\}\right) \chi_{N}\left(z_{1}, \ldots, z_{N} \mid\{\mu\}\right) .
$$

Apply integration by parts; due to (2.2) there is no contribution from the boundary because $\lambda_{j}-\mu_{k} \neq 0$. So we have for the form factor

$$
F(x)=\frac{(2 i)^{N}}{N !} \exp \left\{i x\left(\sum_{j=1}^{N} \mu_{j}-\sum_{j=1}^{N+1} \lambda_{j}\right)\right\} \sum_{P, Q}(-1)^{[P+Q]} \prod_{j=1}^{N} \frac{1}{\lambda_{p_{j}}-\mu_{Q_{j}}} .
$$

Here $Q$ is a permutation of $\left\{\mu_{j}\right\}$ (in number $N$ ) and $P$ is a permutation of $\left\{\lambda_{k}\right\}$ (in number $N+1$ ). Let us remind the reader that $F(x)$ is antisymmetric in $\lambda_{j}$ and in $\mu_{k}$ separately. This allows us to prove the following formula

$$
\frac{1}{N !} \sum_{P, Q}(-1)^{[P+Q]} \prod_{j=1}^{N} \frac{1}{\lambda_{p_{j}}-\mu_{Q_{j}}}=\left.\left(1+\frac{\partial}{\partial d}\right) \operatorname{det}_{N}\left[\frac{1}{\lambda_{j}-\mu_{k}}-\frac{\alpha}{\lambda_{N+1}-\mu_{k}}\right]\right|_{\alpha=0} .
$$

Differentiating the rows one by one, the right-hand side can be represented in the form of $N+1$ terms. The first is $\operatorname{det}\left(1 /\left(\lambda_{j}-\mu_{k}\right)\right)$ all other terms can be obtained from this determinant by the interchange $\lambda_{m} \leftrightarrow \lambda_{N+1}$ which changes the sign. The left-hand side of (2.6) can be represented in a similar form if we group the terms in the following way. First set $\lambda_{p_{N+1}}=\lambda_{N+1}$ : the sum of all such terms is equal to $\operatorname{det}\left(1 /\left(\lambda_{j}-\mu_{k}\right)\right)$, then set $\lambda_{p_{N+1}}=\lambda_{m}$ and collect all the terms. In this way it is clear that formula (2.6) is correct. Another important property is that the right-hand side of (2.6) can be obtained from the product $\prod_{j=1}^{N}\left(\lambda_{j}-\mu_{j}\right)^{-1}$ by means of antisymmetrisation with respect to $\left\{\lambda_{j}\right\}$; explicitly we have

$$
\left.\left(1+\frac{\partial}{\partial \alpha}\right) \operatorname{det}_{N}\left[\frac{1}{\lambda_{j}-\mu_{k}}-\frac{\alpha}{\lambda_{N+1}-\mu_{k}}\right]\right|_{\alpha=0}=\underset{\{\lambda\}}{\operatorname{antisym}} \prod_{j=1}^{N} \frac{1}{\lambda_{j}-\mu_{j}} .
$$

The final result is

$$
\begin{aligned}
F(x) & =\left.(2 i)^{N} \exp \left\{i x\left(\sum_{j=1}^{N} \mu_{j}-\sum_{j=1}^{N+1} \lambda_{j}\right)\right\}\left(1+\frac{\partial}{\partial \alpha}\right) \operatorname{det}_{N} M_{j k}\right|_{\alpha=0}, \\
M_{j k} & =\frac{1}{\lambda_{j}-\mu_{k}}-\frac{\alpha}{\lambda_{N+1}-\mu_{k}} .
\end{aligned}
$$

It is interesting to note that $\operatorname{det} M$ is a linear function of $\alpha$. This follows from the fact that for the ground state

$$
\sum_{j=1}^{N} \mu_{j}=0
$$

The Quantum Bose field depends on time $t$ in the canonical way:

$$
\psi(x, t)=e^{i H t} \psi(x, 0) e^{-i H t} .
$$

So the time dependent form factor is equal to 


$$
\begin{aligned}
F(x, t)= & \left\langle\Psi_{N+1}\{\lambda\}\left|\psi^{+}(x, t)\right| \Psi_{N}\{\mu\}\right\rangle \\
= & \exp \left\{-i h t+i t\left(\sum_{j=1}^{N+1} \lambda_{j}^{2}-\sum_{j=1}^{N} \mu_{j}^{2}\right)+i x\left(\sum_{j=1}^{N} \mu_{j}-\sum_{j=1}^{N+1} \lambda_{j}\right)\right\} \\
& \left.\cdot(2 i)^{N}\left(1+\frac{\partial}{\partial \alpha}\right) \operatorname{det}_{N} M_{j k}\right|_{\alpha=0} .
\end{aligned}
$$

\section{Time Correlator}

Let us decompose the correlator over a complete set of states:

$$
\begin{aligned}
&\left\langle\psi\left(x_{2}, t_{2}\right) \psi^{+}\left(x_{1}, t_{1}\right)\right\rangle= \sum_{\{\lambda\}} \frac{1}{L^{2 N+1}} F^{*}\left(x_{2}, t_{2}\right) F\left(x_{1}, t_{1}\right)= \\
&= \sum_{\{\lambda\}} \exp \left\{i h t_{21}+i t_{21}\left(\sum_{j=1}^{N} \mu_{j}^{2}-\sum_{j=1}^{N+1} \lambda_{j}^{2}\right)\right. \\
&\left.+i x_{12}\left(\sum_{j=1}^{N} \mu_{j}-\sum_{j=1}^{N+1} \lambda_{j}\right)\right\} \cdot \frac{2^{2 N}}{L^{2 N+1}}\left(1+\frac{\partial}{\partial \alpha}\right) \\
&\left.\cdot \operatorname{det}_{N}\left(\frac{1}{\lambda_{j}-\mu_{k}}-\frac{\alpha}{\lambda_{N+1}-\mu_{k}}\right)\right|_{\alpha=0}\left(1+\frac{\partial}{\partial \beta}\right) \\
&\left.\cdot \operatorname{det}_{N}\left(\frac{1}{\lambda_{j}-\mu_{k}}-\frac{\beta}{\lambda_{N+1}-\mu_{k}}\right)\right|_{\beta=0} \cdot
\end{aligned}
$$

Here we use Eq. (2.11), and $x_{12}=x_{1}-x_{2}, t_{21}=t_{2}-t_{1}$. Each of the determinants here are antisymmetric in $\left\{\lambda_{j}\right\}$. Taking into account $(2.7)$, let us replace the second determinant by $\prod_{j=1}^{N} 1 /\left(\lambda_{j}-\mu_{j}\right)$;

$$
\begin{aligned}
\left\langle\psi\left(x_{2}, t_{2}\right) \psi^{+}\left(x_{1}, t_{1}\right)\right\rangle= & (N+1) ! \exp \left\{i h t_{21}+i t_{21} \sum_{j=1}^{N} \mu_{j}^{2}+i x_{12} \sum_{j=1}^{N} \mu_{j}\right\} \\
& \cdot \frac{1}{L}\left(\frac{2}{L}\right)^{2 N} \sum_{\{\lambda\}}\left[e^{i x_{21} \lambda_{N+1}} e^{i t_{12} \lambda_{N+1}^{2}}+\frac{\partial}{\partial \alpha}\right] \\
& \left.\cdot \operatorname{det}_{N}\left[\frac{e^{i t_{12} \lambda_{j}^{2}+i x_{21} \lambda_{j}}}{\left(\lambda_{j}-\mu_{k}\right)\left(\lambda_{j}-\mu_{j}\right)}-\alpha \frac{e^{i t_{12} \lambda_{j}^{2}+i x_{21} \lambda_{j}} e^{i t_{12} \lambda_{N+1}^{2}+i x_{21} \lambda_{N+1}}}{\left(\lambda_{j}-\mu_{j}\right)\left(\lambda_{N+1}-\mu_{k}\right)}\right]\right|_{\alpha=0} .
\end{aligned}
$$

Now replace summation with respect to the sets $\left\{\lambda_{j}\right\}$ by independent summation of each individual $\lambda_{j}$;

$$
(N+1) ! \sum_{\left\{\lambda_{j}\right\}}=\sum_{\lambda_{1}} \sum_{\lambda_{2}} \cdots \sum_{\lambda_{N+1}}
$$

Each of $\lambda_{j}$ run through all the values $(2.3)(2 \pi / L) j / L$ independently. Notice that $\lambda_{j}$ enter only row number $j$ in the determinant (3.2) $j=1, \ldots, N$. So we have 


$$
\begin{aligned}
\left\langle\psi\left(x_{2}, t_{2}\right) \psi^{+}\left(x_{1}, t_{1}\right)\right\rangle= & \exp \left\{i h t_{21}+i t_{21} \sum_{j=1}^{N} \mu_{j}^{2}+i x_{12} \sum_{j=1}^{N} \mu_{j}\right\} \\
& \cdot\left\{\frac{1}{L} \sum_{\lambda_{N+1}} e^{i x_{21} \lambda_{N+1}} e^{i t_{12} \lambda_{N+1}^{2}}+\frac{\partial}{\partial \alpha}\right\} \operatorname{det}_{N}\left[\sum_{\lambda_{j}=2 \pi j / L}\left(\frac{2}{L}\right)^{2}\right. \\
& \cdot\left\{\frac{e^{i t_{12} \lambda_{j}^{2}+x_{21} \lambda_{j}}}{\left(\lambda_{j}-\mu_{k}\right)\left(\lambda_{j}-\mu_{j}\right)}-\alpha \frac{e^{i t_{12} \lambda_{j}^{2}+i x_{21} \lambda_{j}}}{\left(\lambda_{j}-\mu_{j}\right)}\right. \\
& \left.\cdot \frac{1}{L} \sum_{\lambda_{N+1}} \frac{e^{i t_{12} \lambda_{N+1}^{2}+i x_{21} \lambda_{N+1}}}{\left(\lambda_{N+1}-\mu_{k}\right)}\right\}\left.\right|_{\alpha=0}
\end{aligned}
$$

Now let us calculate the sum with respect to the individual $\lambda_{j}$. In Appendix A it is shown that in the thermodynamic limit we have:

$$
\frac{1}{L} \sum_{\lambda_{j}} \frac{e^{i t \lambda_{j}^{2}-i x \lambda_{j}}}{\lambda_{j}-\mu_{k}}=\frac{1}{2 \pi} \int_{-\infty}^{\infty} \frac{d \lambda e^{i t \lambda^{2}-i x \lambda}}{\lambda-\mu_{k}} .
$$

Introduce the notation

$$
E(\lambda \mid t, x)=f_{-\infty}^{\infty} \frac{d \mu e^{i t \mu^{2}-i x \mu}}{\lambda-\mu},
$$

then it is shown in Appendix A that, in thermodynamic limit:

$$
\frac{4}{L^{2}} \sum_{\lambda_{j}} \frac{e^{t t \lambda_{j}^{2}-i x \lambda_{j}}}{\left(\lambda_{j}-\mu_{k}\right)^{2}}=e^{i t \mu_{k}^{2}-i x \mu_{k}}+\frac{2 \pi}{L} \frac{\partial}{\partial \mu_{k}} E\left(\mu_{k} \mid t, x\right) .
$$

One should also remember that

$$
\frac{1}{L} \sum_{\lambda_{j}} e^{i t \lambda_{j}^{2}-i x \dot{\lambda}_{j}}=\frac{1}{2 \pi} \int_{-\infty}^{\infty} e^{i t \lambda^{2}-i x \lambda} d \lambda .
$$

We introduce also another notation:

$$
G(t, x)=\int_{-\infty}^{\infty} e^{i t \mu^{2}-i x \mu} d \mu .
$$

Using all these summation formulae we may write the determinant formula (3.4) for the correlation function in the form

$$
\begin{aligned}
\left\langle\psi\left(x_{2}, t_{2}\right) \psi^{+}\left(x_{1}, t_{1}\right)\right\rangle= & e^{i h t_{21}}\left(\frac{1}{2 \pi} G\left(t_{12}, x_{12}\right)+\frac{\partial}{\partial \alpha}\right) \\
& \cdot \operatorname{det}_{N}\left(\delta_{j k}+\exp \left[i t_{2} \mu_{j}^{2}-i x_{2} \mu_{j}-i t_{1} \mu_{k}^{2}+i x_{1} \mu_{k}\right]\right. \\
& \cdot\left[\frac{2}{\pi L\left(\mu_{j}-\mu_{k}\right)}\left(E\left(\mu_{j} \mid t_{12}, x_{12}\right)-E\left(\mu_{k} \mid t_{12}, x_{12}\right)\right)\right. \\
& \left.\left.-\frac{\alpha}{L \pi^{2}} E\left(\mu_{j} \mid t_{12}, x_{12}\right) E\left(\mu_{k} \mid t_{12}, x_{12}\right)\right]\right)\left.\right|_{\alpha=0} .
\end{aligned}
$$


Calculation of the thermodynamic limit of this result is straightforward. The final result for the zero temperature correlator is

$$
\left\langle\psi\left(x_{2}, t_{2}\right) \psi^{+}\left(x_{1}, t_{1}\right)\right\rangle=\left.e^{i h t_{21}}\left(\frac{1}{2 \pi} G\left(t_{12}, x_{12}\right)+\frac{\partial}{\partial \alpha}\right) \operatorname{det}\left(1+V_{0}\right)\right|_{\alpha=0} .
$$

Here $V_{0}$ is an integral operator, acting on the interval $[-q, q]$. The kernel of this operator $V_{0}(\lambda, \mu)$ is equal to

$$
\begin{aligned}
V_{0}(\lambda, \mu)= & {\left[\frac{E\left(\lambda \mid t_{12}, x_{12}\right)-E\left(\mu \mid t_{12}, x_{12}\right)}{\pi^{2}(\lambda-\mu)}-\frac{\alpha}{2 \pi^{3}} E\left(\lambda \mid t_{12}, x_{12}\right) E\left(\mu \mid t_{12}, x_{12}\right)\right] } \\
& \cdot \exp \left(\frac{i}{2} t_{21}\left(\lambda^{2}+\mu^{2}\right)-\frac{i}{2} x_{21}(\lambda+\mu)\right) .
\end{aligned}
$$

This is our final answer for time correlator at zero temperature.

Now let us consider the equal time case: $t_{1}=t_{2}$.

In this case $(1 / 2 \pi) G\left(0, x_{12}\right)=\delta\left(x_{1}-x_{2}\right)$; this gives commutators

$$
\begin{gathered}
{\left[\psi\left(x_{1}\right), \psi^{+}\left(x_{2}\right)\right]=\delta\left(x_{1}-x_{2}\right),} \\
E\left(\mu \mid 0, x_{12}\right)=-i \pi e^{-i \mu x_{12}} \text { for } \chi_{12}>0 .
\end{gathered}
$$

Also, the kernel $V_{0}$ is reduced to

$$
V_{0}(\lambda, \mu)=-\frac{2}{\pi} \frac{\sin \left(\left(x_{12} / 2\right)(\lambda-\mu)\right)}{(\lambda-\mu)}+\frac{\alpha}{2 \pi} e^{i \lambda x_{21} / 2} e^{i \mu x_{12} / 2} .
$$

In Appendix $B$ it is shown that Eqs. (3.13), (3.11) are equivalent to the zero temperature version of the Lenard formula for equal time correlators.

\section{Temperature Time Correlator}

Now let us discuss the temperature correlator

$$
\left\langle\psi\left(x_{2}, t_{2}\right) \psi^{+}\left(x_{1}, t_{1}\right)\right\rangle_{T}=\frac{\operatorname{tr}\left\{\psi\left(x_{2}, t_{2}\right) \psi^{+}\left(x_{1}, t_{1}\right) e^{-H / T}\right\}}{\operatorname{tr}\left(e^{-H / T}\right)} .
$$

We shall use Euclidean time, $\tau=i t$. Evolution in $\tau$ is given by

$$
\psi(x, \tau)=e^{H \tau} \psi(x, 0) e^{-H \tau} .
$$

Euclidean temperature correlators are defined in a similar way to (4.1)

$$
\left\langle\psi\left(x_{2}, \tau_{2}\right) \psi^{+}\left(x_{1}, \tau_{1}\right)\right\rangle_{T}=\frac{\operatorname{tr}\left\{\psi\left(x_{2}, \tau_{2}\right) \psi^{+}\left(x_{1}, \tau_{1}\right) e^{-H / T}\right\}}{\operatorname{tr}\left(e^{-H / T}\right)} .
$$

The traces entering (4.1) and (4.2) can be represented as functional integrals [6], which can be evaluated by the method of steepest descent in the thermodynamic limit. Only states of thermodynamic equilibrium contribute. They are the set of eigenfunctions of the Hamiltonian (an infinite number in the thermodynamic limit), which were described completely by C. N. Yang and C. P. Yang [3]. All these eigenfunctions correspond to the same distribution function in momentum space 
(1.8). It is interesting to mention that the mean value of $\psi\left(x_{2}, \tau_{2}\right) \psi^{+}\left(x_{1}, \tau_{1}\right)$ with respect to one of these eigenfunctions is independent of the choice of eigenfunction (if it belongs to the set of states of thermal equilibrium). This mean value is equal to the correlator (4.2). This permits us to calculate the temperature correlator (4.2) by means of formula (3.10)

$$
\left\langle\psi\left(x_{2}, \tau_{2}\right) \psi^{+}\left(x_{1}, \tau_{1}\right)\right\rangle_{T}=e^{h \tau_{21}}\left(\frac{1}{2 \pi} G\left(\tau_{12}, x_{1}\right)+\frac{\partial}{\partial \alpha}\right) \times\left.\operatorname{det}(1+V)\right|_{\alpha=0} .
$$

Here $V$ is integral operator acting on the real axis. The kernel $V(\lambda, \mu)$ being equal to

$$
\begin{aligned}
V(\lambda, \mu)= & \frac{e^{(1 / 2) \tau_{21}\left(\lambda^{2}+\mu^{2}\right)} e^{-(i / 2) x_{21}(\lambda+\mu)}}{\sqrt{1+e^{\left(\lambda^{2}-h\right) / T}} \sqrt{1+e^{\left(\mu^{2}-h\right) / T}}} \\
& \cdot\left[\frac{E\left(\lambda \mid \tau_{12}, x_{12}\right)-E\left(\mu \mid \tau_{12}, x_{12}\right)}{\pi^{2}(\lambda-\mu)}-\frac{\alpha}{2 \pi^{3}} E\left(\lambda \mid \tau_{12}, x_{12}\right) E\left(\mu \mid \tau_{12}, x_{12}\right)\right],
\end{aligned}
$$

where $x_{12}=x_{1}-x_{2} ; 0 \leqq \tau_{21}=\tau_{2}-\tau_{1} \leqq 1 / T ; \tau_{12}=-\tau_{21}$. Also, we used the notation

$$
\begin{gathered}
G\left(\tau_{12}, x_{12}\right)=\int d \mu e^{-\tau_{21} \mu^{2}-i x_{12} \mu}, \\
E\left(\mu \mid \tau_{12}, x_{12}\right)=f \frac{d \lambda}{\lambda-\mu} e^{-\tau_{21} \lambda^{2}-i x_{12} \lambda} .
\end{gathered}
$$

This is the final result for the time temperature correlator in the Euclidean case. It is represented as a Fredholm minor.

Now let us present a similar representation for the real time correlator (4.1). It can be obtained from the above formulae by the replacement $\tau=i t$, or from the zero temperature case (3.11) by replacement of $V_{0}$ by $V$, acting on the whole real axis. The kernel $V(\lambda, \mu)$ is equal to

$$
V(\lambda, \mu)=\frac{V_{0}(\lambda, \mu)}{\sqrt{1+e^{\left(\lambda^{2}-h\right) / T}} \sqrt{1+e^{\left(\mu^{2}-h\right) / T}}} .
$$

For $V_{0}(\lambda, \mu)$ see $(3.12)$

$$
\left\langle\psi\left(x_{2}, t_{2}\right) \psi^{+}\left(x_{1} t_{1}\right)\right\rangle_{T}=\left.e^{i h \tau_{21}}\left(\frac{1}{2 \pi} G\left(t_{12}, x_{1}\right)+\frac{\partial}{\partial \alpha}\right) \operatorname{det}(1+V)\right|_{\alpha=0} .
$$

Here $G$ is given by (3.9). The equal time case is treated similarly to the zero temperature case; now we need

$$
V(\lambda, \mu)=\frac{V_{0}(\lambda, \mu)}{\sqrt{1+e^{\left(\lambda^{2}-h\right) / T}} \sqrt{1+e^{\left(\mu^{2}-h\right) / T}}} .
$$

Here $V_{0}(\lambda, \mu)$ is given by (3.13). In Appendix B it is shown that the Fredholm minor of (4.9) (as the formula for $\left\langle\psi\left(x_{2}\right) \psi^{+}\left(x_{1}\right)\right\rangle_{T}$ ) is equivalent to the Lenard formula.

\section{Conclusion}

We have represented the time-space dependent field correlator of an impenetrable Bose gas as a Fredholm minor. The case of many field correlators will be considered 
by N. A. Slavnov in a forthcoming paper. In our next publication we shall present differential equations driving these correlation functions. These will be generalizations of Painlevé equations to partial differential equations.

\section{Appendix A}

First let us study the behaviour in the thermodynamic limit of the following sums:

$$
S_{1}=\sum_{\lambda_{j}=2 \pi j / L} \frac{g\left(\lambda_{j}\right)}{\lambda_{j}-\mu_{k}} .
$$

Here $j$ runs through all integer (positive and negative) and $g(\lambda)$ is a smooth decreasing function, for example $g(\lambda)=\exp \left(i t \lambda^{2}-i x \lambda\right)$. (We shall use the standard regularisation $t \rightarrow t+i 0$.) We transform (A.1),

$$
\begin{aligned}
S_{1} & =\frac{1}{L} \sum_{\lambda_{j}=2 \pi j / L} \frac{g\left(\lambda_{j}\right)-g\left(\mu_{k}\right)}{\lambda_{j}-\mu_{k}}+\frac{1}{2 \pi} g\left(\mu_{k}\right) \sum_{j \in Z} \frac{1}{j-\frac{1}{2}} \\
& =\frac{1}{2 \pi} \int \frac{d \lambda}{\lambda-\mu_{k}}\left(g(\lambda)-g\left(\mu_{k}\right)\right)=\frac{1}{2 \pi} \int \frac{d \lambda}{\lambda-\mu_{k}} g(\lambda) .
\end{aligned}
$$

Here we used the formulae

$$
\sum_{j \in Z} \frac{1}{j-\frac{1}{2}}=0 ; \quad f \frac{d \lambda}{\lambda-\mu}=0 .
$$

So the thermodynamic limit of the sum (A.1) is equal to the integral (A.2)

$$
\frac{1}{L} \sum_{\lambda_{j}=2 \pi j / L} \frac{g\left(\lambda_{j}\right)}{\lambda_{j}-\mu_{k}} \rightarrow \frac{1}{2 \pi} f \frac{d \lambda}{\lambda-\mu_{k}} g(\lambda) .
$$

Consider now the more singular sum:

$$
S_{2}=\frac{1}{L} \sum_{\lambda_{j}=2 \pi j / L} \frac{g\left(\lambda_{j}\right)}{\left(\lambda_{j}-\mu_{k}\right)^{2}} .
$$

To study the thermodynamic limit, we transform it in a similar way:

$$
S_{2}=\frac{g\left(\mu_{k}\right)}{L} \sum_{\lambda_{j}}\left(\frac{1}{\lambda_{j}-\mu_{k}}\right)^{2}+\frac{1}{L} \sum_{\lambda_{j}} \frac{g\left(\lambda_{j}\right)-g\left(\mu_{k}\right)}{\left(\lambda_{j}-\mu_{k}\right)^{2}} .
$$

The first term can be transformed in the following way:

$$
\frac{g\left(\mu_{k}\right)}{L} \sum_{\lambda_{j}}\left(\frac{1}{\lambda_{j}-\mu_{k}}\right)^{2}=\frac{L}{4 \pi^{2}} g\left(\mu_{k}\right) \sum_{j \in Z}\left(\frac{1}{j-\frac{1}{2}}\right)^{2}=\frac{L}{4} g\left(\mu_{k}\right) .
$$

The second term can be written, by means of (A.4), in the form

$$
\frac{1}{L} \sum_{\lambda_{j}} \frac{g\left(\lambda_{j}\right)-g\left(\mu_{k}\right)}{\left(\lambda_{j}-\mu_{k}\right)^{2}} \rightarrow \frac{1}{2 \pi} f d \lambda \frac{g\left(\lambda_{j}\right)-g\left(\mu_{k}\right)}{\left(\lambda_{j}-\mu_{k}\right)^{2}} .
$$


Now we use (A.3) to get

$$
\frac{1}{2 \pi} f d \lambda \frac{g\left(\lambda_{j}\right)-g\left(\mu_{k}\right)}{\left(\lambda_{j}-\mu_{k}\right)^{2}}=\frac{1}{2 \pi} \frac{\partial}{\partial \mu_{k}} f d \lambda \frac{g(\lambda)-g\left(\mu_{k}\right)}{\left(\lambda_{j}-\mu_{k}\right)}=\frac{1}{2 \pi} \frac{\partial}{\partial \mu_{k}} f \frac{d \lambda \cdot g(\lambda)}{\lambda-\mu_{k}} .
$$

Finally we have

$$
\frac{1}{L} \sum_{\lambda_{j}=2 \pi j / L} \frac{g\left(\lambda_{j}\right)}{\left(\lambda_{j}-\mu_{k}\right)^{2}} \rightarrow \frac{L}{4} g\left(\mu_{k}\right)+\frac{1}{2 \pi} \frac{\partial}{\partial \mu_{k}} f \frac{d \lambda}{\lambda-\mu_{k}} g(\lambda) .
$$

In our case we obtain (3.7)

$$
\sum_{\lambda_{j}} \frac{e^{i t \lambda_{j}^{2}-i x \lambda_{j}}}{\left(\lambda_{j}-\mu_{k}\right)^{2}}=\frac{L^{2}}{4} e^{i t \mu_{k}^{2}-i \times \mu_{k}}+\frac{L}{2 \pi} \frac{\partial}{\partial \mu_{k}} E\left(\mu_{k} \mid t, x\right) .
$$

\section{Appendix B}

For the equal temperature correlator Lenard [1] showed that

$$
\left\langle\psi^{+}(x) \psi(-x)\right\rangle_{T}=\frac{1}{\pi} \rho(x,-x) \operatorname{det}\left(1-\frac{2}{\pi} \Theta\right) ; \quad x \geqq 0 .
$$

Here we use the fact that $\left\langle\psi^{+}\left(x_{1}\right) \psi\left(x_{2}\right)\right\rangle$ depends only on difference $x_{1}-x_{2}$. In (B.1) $\theta$ is a linear integral operator acting on the interval $[-x, x]$ with the kernel

$$
\Theta(\xi-\eta)=\frac{1}{2} \int_{-\infty}^{\infty} d \lambda e^{i(\xi-\eta) \lambda} \theta(\lambda)
$$

Here we denote

$$
\theta(\lambda)=\frac{1}{1+e^{\left(\lambda^{2}-h\right) / T}}
$$

The kernel of the resolvents operator is defined in the usual way:

$$
\rho(\xi, \eta)-\frac{2}{\pi} \int_{-x}^{x} \Theta\left(\xi-\xi^{\prime}\right) \rho\left(\xi^{\prime}, \eta\right) d \xi^{\prime}=\Theta(\xi-\eta) .
$$

The value of the kernel $\rho(x,-x)$ at the ends of the interval $[-x, x]$ enter (B.1).

Our formula (3.13), (4.9) for the equal time case can be represented in the following way:

$$
\left\langle\psi^{+}(x) \psi(-x)\right\rangle_{T}=\frac{1}{2 \pi} \operatorname{det}\left(1-\frac{2}{\pi} K\right) \cdot \int d \lambda e^{i \lambda x} \sqrt{\theta(\lambda)} f_{+}(\lambda) .
$$

Here we evaluate the derivative with respect to $\alpha$. In (B.5) $K$ is the integral operator on the real axis with the kernel

$$
K(\lambda, \mu)=\sqrt{\theta(\lambda)} \frac{\sin x(\lambda-\mu)}{(\lambda-\mu)} \sqrt{\theta(\mu)},
$$


and the function $f(\lambda)$ is defined by the integral equation

$$
f_{ \pm}(\lambda)-\frac{2}{\pi} \int_{-\infty}^{\infty} K(\lambda, \mu) f_{ \pm}(\mu) d \mu=e^{ \pm i \lambda x} \sqrt{\theta(\lambda)}
$$

We will show, by means of Fourier transformation, that (B.1) and (B.7) are equivalent. Consider the integral equation

$$
\varphi(\xi)-\frac{2}{\pi} \int_{-x}^{x} \Theta\left(\xi-\xi^{\prime}\right) \varphi\left(\xi^{\prime}\right) d \xi^{\prime}=Q(\Phi)
$$

Taking the following Fourier transforms

$$
\begin{aligned}
& \varphi(\xi)=\int_{-\infty}^{\infty} d \lambda \sqrt{\theta(\lambda)} e^{-i \lambda \xi} f(\lambda), \\
& f(\lambda)=\frac{1}{2 \pi \sqrt{\theta(\lambda)}} \int_{-\infty}^{\infty} d \xi e^{i \lambda \xi} \Phi(\xi), \\
& F(\lambda)=\frac{1}{2 \pi \sqrt{\theta(\lambda)}} \int_{-\infty}^{\infty} d \xi e^{i \lambda \xi} \Phi(\xi),
\end{aligned}
$$

one has the following equation for the function $f(\lambda)$ :

$$
f(\lambda)-\frac{2}{\pi} \int_{-\infty}^{\infty} K(\lambda, \mu) f(\mu) d \mu=F(\lambda)
$$

The kernel $K(\lambda, \mu)$ is exactly (B.6). So the Fredholm determinants of operators (B.2) and (B.5) are the same:

$$
\operatorname{det}\left(1-\frac{2}{\pi} K\right)=\operatorname{det}\left(1-\frac{2}{\pi} \theta\right)
$$

Now let us show that

$$
\rho(x,-x)=\frac{1}{2} \int_{-\infty}^{\infty} e^{i \lambda x} \sqrt{\theta(\lambda)} f_{+}(\lambda) d \lambda=\frac{1}{2} \int_{-\infty}^{\infty} e^{i \lambda x} \sqrt{\theta(\lambda)} f_{-}(\lambda) d \lambda .
$$

Here we change the sign of the integration variable $\lambda$. To prove (B.12), consider Eq. (B.4) for $\eta=-x$. The Fourier transform of $\rho(\xi,-x)$ is denoted by $r(\lambda)$,

$$
r(\lambda)=\frac{1}{2 \pi \sqrt{\theta(\lambda)}} \int_{-\infty}^{\infty} d \xi e^{i \lambda \xi} \rho(\xi,-x) .
$$

Fourier transformation of (B.4) leads to the equation for $r(\lambda)$,

$$
r(\lambda)-\frac{2}{\pi} \int K(\lambda, \mu) r(\mu) d \mu=\frac{1}{2} \sqrt{\theta(\lambda)} e^{-i x \lambda} .
$$

Comparison with (B.7) shows that

$$
r(\lambda)=\frac{1}{2} f_{-}(\lambda)
$$


Inverse Fourier transformation shows that

$$
\rho(x,-x)=\frac{1}{2} \int_{-\infty}^{\infty} d \lambda e^{i \lambda x} \sqrt{\theta(\lambda)} f_{-}(\lambda)
$$

So (B.12) is proved. This finally shows that (B.1) and (B.5) are equivalent. Also, our formula in the equal time case is equivalent to the Lenard formula [1].

\section{References}

1. Lenard, A.: One-dimensional impenetrable bosons in thermal equilibrium. J. Math. Phys. 7, 1268-1272 (1966)

2. Lieb, E. H., Mattis, D. C. (eds.): Mathematical physics in one dimension. New York:, London: Academic Press 1966

3. Yang, C. N., Yang, C. P.: Thermodynamics of a one-dimensional system of bosons with repulsive delta-function interaction. J. Math. Phys. 10, 1115-1122 (1969)

4. Jimbo, M., Miwa, T., Mori, Y., Sato, M.: Density matrix of an impenetrable Bose gas and the fifth Painlevé transcendent. Physica 1D, 80-158 (1980)

5. Its, A. R., Izergin, A. G., Korepin, V. E.: Completely integrable differential equations for temperature correlation functions of an impenetrable Bose gas, submitted to CMP, Preprint. International Centre for Theoretical Physics, Miramare-Trieste (1989), IC/89/139

6. Bogoliubov, N. M., Izergin, A. G., Korepin, V. E.: Quantum inverse scattering method and correlation functions. Shastry, B. S., Jha, S. S., Singh, V. (eds.): Lecture Notes in Physics, vol. 242, p. 243. Berlin, Heidelberg, New York: Springer 1985

Communicated by A. Jaffe

Received August 17, 1989 
